Check for updates

Cite this: J. Mater. Chem. C, 2020 8, 12886

Received 2nd June 2020

Accepted 12th August 2020

DOI: $10.1039 / \mathrm{d0tc02637a}$

rsc.li/materials-c

\title{
Influence of mechanical, thermal, and electrical perturbations on the dielectric behaviour of guest-encapsulated HKUST-1 crystals $\dagger$
}

\author{
Arun Singh Babal and Jin-Chong Tan (D) *
}

\begin{abstract}
Metal-organic frameworks (MOFs) are emerging low- $k$ dielectric materials for application in next-generation microelectronics and telecommunication devices. MOF dielectrics can function as smart sensors with high sensitivity and chemical selectivity, by leveraging the ubiquitous dielectric response of MOFs and overcoming the limitations of DC conductivity and fluorescence approaches. Herein we study the effects of material synthesis, applied mechanical stress (37-520 MPa), varying temperature $\left(20-100{ }^{\circ} \mathrm{C}\right)$, and guest encapsulation on the frequency-dependent dielectric response $(4 \mathrm{~Hz}$ to $1 \mathrm{MHz})$ and $\mathrm{AC}$ conductivity of the HKUST-1 MOF. Particularly, we show that the confinement of the triethylamine $\left(\mathrm{NEt}_{3}\right)$ guest molecules in HKUST-1 (host) yields a NEt 3 aHKUST-1 system that is tuneable via mechanical, thermal and electrical perturbations. Within the frequency range of $10 \mathrm{kHz}$ to $1 \mathrm{MHz}$, at $20{ }^{\circ} \mathrm{C}$, we show that the dielectric constant $\left(\varepsilon^{\prime}\right)$ of the guest-encapsulated system could be tuned to attain a value in the range of 2.8 to 7.2 ; at $100{ }^{\circ} \mathrm{C}$, an even greater value of $\varepsilon^{\prime}$ in the range of 3.1 to 9.5 could be achieved. Conversely, we found that the dielectric tuneability of the porous (guest-free) HKUST-1 is relatively more limited $\left(\varepsilon^{\prime}=2.8\right.$ to 4.9$)$ whilst employing the same operational parameters. Furthermore, the confinement of guest molecules in HKUST-1 enhances the mechanical resilience and yield strength of the powders subject to a compressive pelleting stress. Together, the results elucidate the new potential for exploiting host-guest interactions in MOFs, coupled with electrical, thermal, and mechanical stimuli to regulate the precise dielectric response of a designer low- $k$ material.
\end{abstract}

\section{Introduction}

In recent years, the research on low- $k$ dielectric materials has gained attention due to their potential for enhancing the performance of next-generation electronic devices and telecommunications. Metal-organic frameworks (MOFs) are highly porous hybrid materials consisting of organic linkers connected to inorganic metal sites to yield tuneable crystalline materials. ${ }^{1-3}$ Their unique properties such as ordered framework structures having long-range porosity, low density and high thermal/chemical stability make them a potential candidate for the interlayer dielectric applications. Electronic device miniaturisation has introduced new challenges such as higher RC time delay, crosstalks between the circuits, and power consumption penalty. ${ }^{4,5}$ The aforementioned challenges can be addressed by implementing new ultralow- $k$ dielectric materials.

Multifunctional Materials and Composites (MMC) Laboratory, Department of Engineering Science, University of Oxford, Parks Road, Oxford, OX1 3PJ, UK.

E-mail: jin-chong.tan@eng.ox.ac.uk

$\dagger$ Electronic supplementary information (ESI) available. See DOI: 10.1039/ d0tc02637a
Theoretical calculations of MOF-based dielectrics have predicted hypothetical static dielectric constant values of less than 2.0, ${ }^{6,7}$ which are well below the conventionally used $\mathrm{SiO}_{2}$ whose static dielectric constant is $k \sim 4$.

While the studies concerning the dielectric properties of MOFs are quite limited compared to their research pertaining to adsorption/desorption and separations, the nascent field of MOF dielectrics is steadily growing. For example, we have studied the dielectric properties of a commercial grade of HKUST-1 powder [ $\mathrm{Cu}_{3} \mathrm{BTC}_{2}$; BTC = benzene-1,3,5-tricarboxylate] in the broadband frequency region ( $\mathrm{Hz}$ and $\mathrm{THz}$ ). The work has revealed all the dielectric transitions as a function of pelleting pressure, and elucidated a number of polarisation mechanisms in HKUST-1. ${ }^{8}$ More recently, we characterised the transient dielectric response of a single crystal of HKUST-1 by subjecting it to different guest environments. ${ }^{9}$ Other earlier exemplars include Lu et al. who synthesized two different Ni-based supramolecular compounds that possess different types of polar guest molecules. ${ }^{10}$ They reported that the solvent molecule and counterions play an important role in regulating the dielectric property of the resulting material. Usman et al. reported the temperature-dependent dielectric behaviour of a 
strontium-based MOF $\left(\left[\mathrm{Sr}_{2}(1,3-\mathrm{bdc})_{2}\left(\mathrm{H}_{2} \mathrm{O}\right)_{2}\right] \cdot \mathrm{H}_{2} \mathrm{O}\right)_{n}(1,3-\mathrm{bdc}=$ 1,3-bis(4,5-dihydro-2-oxazolyl)benzene). ${ }^{11}$ They reported an increase in dielectric constant with temperature because of the freezing and defreezing of water molecules. The dehydrated sample showed a low dielectric constant value of 2.4 at $1 \mathrm{MHz}$ frequency and thermally stable up to $420{ }^{\circ} \mathrm{C}$. Lu et al. also reported a guest-free and solvent resistant $\mathrm{Zn}$-based MOF $\left[\mathrm{Zn}_{2}(\text { Hbbim })_{2}(\text { bbim })\right]_{n}\left(\mathrm{H}_{2}\right.$ bbim $=$ bisbenzimidazole $) .{ }^{12}$ They showed a low dielectric constant of 3.05 at $1 \mathrm{MHz}$, which was found to be stable in the temperature range tested $(3.5-350 \mathrm{~K})$.

MOF materials can be tuned by different synthesis methods, ${ }^{13}$ controlled reaction parameters ${ }^{14}$ and modulators. ${ }^{15}$ Using a modulator is an effective way of controlling the size and morphology of MOF crystals. Xin et al. studied the effects of modulators (sodium acetate and triethylamine $\left(\mathrm{NEt}_{3}\right)$ ) and the type of copper source on the structure and morphology of the resulting HKUST-1. ${ }^{16}$ They showed that the effect of the modulator varies according to the copper system and it depends on the nucleation rate.

In this work, we have employed two synthesis routes to yield a porous and guest-encapsulated HKUST-1 MOF crystal exhibiting different morphologies. The dielectric response of the guestencapsulated material is contrasted against that of the porous guest-free framework by imposing a range of stimuli, such as the alternating electric field, temperature variation, and mechanical stress. To the best of our knowledge, this study represents the first example of its kind where the tuneable dielectric response of a MOF material has been systematically probed subject to different physical and chemical stimuli. The results reveal the scope to engineer bespoke MOF dielectrics targeting technological applications ranging from low- $k$ to high- $k$ integrated electronics, sensors, and telecommunication devices.

\section{Experimental section}

\subsection{Materials}

Copper nitrate trihydrate $\left(\mathrm{Cu}\left(\mathrm{NO}_{3}\right)_{2} \cdot 3 \mathrm{H}_{2} \mathrm{O}, 99 \%\right)$, 1,3,5-benzenetricarboxylic acid $\left(\mathrm{H}_{3} \mathrm{BTC}, 98 \%\right)$, methanol $\left(\mathrm{CH}_{3} \mathrm{OH}, 99.5 \%\right)$ and triethylamine $\left(\mathrm{NEt}_{3}, 99 \%\right)$ were purchased from Sigma Aldrich. All the chemicals were used without further purification.

\subsection{Synthesis of HKUST-1 (without using $\mathrm{NEt}_{3}$ )}

The solvothermal synthesis of HKUST-1 was carried out under ambient conditions. Both $\mathrm{Cu}\left(\mathrm{NO}_{3}\right)_{2} \cdot 3 \mathrm{H}_{2} \mathrm{O}(6 \mathrm{~g})$ and $\mathrm{H}_{3} \mathrm{BTC}$ $(2.52 \mathrm{~g})$ were dissolved separately in $150 \mathrm{~mL}$ of $\mathrm{CH}_{3} \mathrm{OH}$ and later on, an additional $100 \mathrm{~mL}$ of $\mathrm{CH}_{3} \mathrm{OH}$ was added into the mixture and stirred for $30 \mathrm{~min}$. The synthesized product was washed several times using methanol to remove excess linkers and then dried overnight in the oven at $80{ }^{\circ} \mathrm{C}$. The yield of the final product was about $10 \%$ with respect to the metal content. The obtained powder sample was denoted as HKUST-1-S.

\subsection{Synthesis of HKUST-1 using NEt $_{3}$}

The lack of precipitation in the standard HKUST-1 synthesis described above was obvious. To improve the product yield, an excess of $\mathrm{NEt}_{3}(6.06 \mathrm{~mL})$ was added to the solution to expedite the deprotonation of the $\mathrm{H}_{3}$ BTC linkers. A blue precipitate comprising nanocrystals of HKUST-1 was formed instantly when the metal and organic linker solutions were combined. ${ }^{17}$ After the reaction (same steps as a standard reaction), the mother liquor was colourless indicating the depletion of copper ions after HKUST-1 synthesis. The yield of the final product was about $90 \%$ with respect to the metal content. The powder sample obtained using this method was designated as HKUST-1-T. This method leads to the encapsulation of $\mathrm{NEt}_{3}$ guest molecules in the HKUST-1 pores.

\subsection{HKUST-1 pellet preparation and density measurements}

The pellets of $13 \mathrm{~mm}$ diameter were prepared under different compressive loads (uniaxial force $=0.5,1,3,5$ to 7 tons) using a hydraulic press (Specac 15 tons). The mass of each pellet was kept constant at $150 \mathrm{mg}$. These pellets were designated as HKUST-1-S- $N$ or HKUST-1-T- $N$, where $N$ is the pelleting load in tons. Subsequently, the nominal density of the pellets was determined from mass/volume.

\subsection{Morphology studies}

The HKUST-1 crystals were characterised using a scanning electron microscope (SEM, Carl Zeiss EVO LS15). The HKUST-1 crystals mounted on the carbon tape were gold sputter coated for $60 \mathrm{~s}$, prior to SEM imaging at $30 \mathrm{keV}$ under high vacuum.

AFM imaging was carried out using the Veeco Dimension 3100 instrument operating under tapping mode, equipped with a Tap300-G probe with a resonant frequency of $300 \mathrm{kHz}$ and a force constant of $40 \mathrm{~N} \mathrm{~m}^{-1}$.

\subsection{Infrared spectroscopy}

FTIR spectra of the powders and pellets were recorded in the mid-IR region (400-4000 $\mathrm{cm}^{-1}$ ) using the Nicolet-iS10 FTIR spectrometer equipped with an ATR sample accessory. The spectral resolution of the instrument was $0.5 \mathrm{~cm}^{-1}$.

\subsection{Thermogravimetric analyses}

Thermogravimetric analysis (TGA) was used to investigate the thermal stability of the HKUST-1 samples. The measurements were performed using the TGA-Q50 machine (TA Instruments) under an inert atmosphere of nitrogen. For this study, the samples were heated from $40{ }^{\circ} \mathrm{C}$ to $500{ }^{\circ} \mathrm{C}$ at $10{ }^{\circ} \mathrm{C} \mathrm{min} \mathrm{m}^{-1}$ in a platinum sample holder.

\subsection{X-Ray diffraction}

$\mathrm{X}$-ray diffraction (XRD) of the pellets was carried out in a transmission mode using the Xenocs NanoInXider machine (using the $\mathrm{Cu} \mathrm{K} \mathrm{K}_{\alpha 1}$ source operating at $30 \mathrm{~W}, \lambda=0.154 \mathrm{~nm}$ ). The XRD spectrum of each pellet was recorded at $2 \theta=4-60^{\circ}$ under a very high-resolution (VHR) beam setting for $2000 \mathrm{~s}$ (scan rate $=0.00357^{\circ} \mathrm{s}^{-1}$, step size $=0.01^{\circ}$ ). The spot size and the flux of the beam were $400 \mu \mathrm{m}$ and $15 \mathrm{Mph} \mathrm{s}^{-1}$, respectively. Before the XRD characterization, pellets were pre-evacuated for $16 \mathrm{~h}$ to mimic the conditions of the samples in dielectric measurements. 


\subsection{Dielectric properties studies}

The electrical properties of HKUST-1 in the frequency range of $4 \mathrm{~Hz}$ to $1 \mathrm{MHz}$ were investigated using the Hioki-IM3536 LCR meter. These measurements were performed in the temperature range of $20-100{ }^{\circ} \mathrm{C}$ with a step size of $10{ }^{\circ} \mathrm{C}$ under a vacuum of $1 \times 10^{-3}$ bar. A thin layer of silver conducting paint (RS) was applied on the opposing sides of the pellets to yield a parallel-plate capacitor configuration. The silver-coated pellets were mounted between two flat and circular aluminium electrodes (13 mm diameter). The setup was calibrated in the vacuum oven to eliminate any parasitic impedance and admittance. All pellets were evacuated in a vacuum $\left(10^{-3}\right.$ bar $)$ for $16 \mathrm{~h}$ beforehand to activate the HKUST-1 framework by removing any moisture content. The real part of dielectric constant $\left(\varepsilon^{\prime}\right)$ and the imaginary part of the dielectric constant $\left(\varepsilon^{\prime \prime}\right)$ were determined using the following equations:

$$
\begin{gathered}
\varepsilon^{\prime}=\frac{C d}{\varepsilon_{0} A} \\
\varepsilon^{\prime \prime}=\varepsilon^{\prime} \tan \delta
\end{gathered}
$$

where $C$ is the capacitance, $d$ is the distance between the pair of parallel plate electrodes, $A$ is the area of the electrode, $\varepsilon_{0}$ is vacuum permittivity and $\tan \delta$ is the loss tangent.

\section{Results and discussion}

\subsection{Effects of $\mathrm{NEt}_{3}$ on the size, morphology, and porosity of HKUST-1 crystals}

The SEM micrograph shown in Fig. 1(a) shows the HKUST-1-S sample comprising crystals with an average size of $1.5 \pm$ $0.7 \mu \mathrm{m}$. However, the morphology of the HKUST-1-T sample is completely different, suggesting the formation of larger aggregates as can be seen in Fig. 1(b). However, using AFM imaging we established that these aggregates are in fact made from nanosized crystals, whose size is $c a .48 \pm 17 \mathrm{~nm}$, as shown in Fig. 1(c and d). Additional SEM and AFM images of the HKUST-1 crystals are given in the ESI $\dagger$ (Fig. S1 and S2). The significant difference in terms of their crystal morphology can be explained by the use of $\mathrm{NEt}_{3}$ in the synthesis of HKUST-1-T, where the rapid deprotonation of $\mathrm{H}_{3} \mathrm{BTC}$ by $\mathrm{NEt}_{3}$ (as a base) has resulted in a high nucleation rate but this suppressed subsequent crystal growth. Upon drying the small nanocrystals will fuse together to yield agglomerates like those found in Fig. 1(b and c).

Fig. 2(a) shows the variation of nominal density as a function of pelleting pressure for the two series of samples prepared from the HKUST-1-S and HKUST-1-T crystals. The density curves for the pellets obey an exponential function of the form $\rho \propto-\exp (-\sigma)$, where $\rho$ and $\sigma$ are the nominal pellet density and nominal stress, respectively. At the highest 7 ton load, the nominal densities of the resulting pellets have risen to $\sim 185 \%$ (HKUST-1-S) and $\sim 200 \%$ (HKUST-1-T) compared with the theoretical density of an ideal HKUST-1 crystal (948.9 $\mathrm{kg} \mathrm{m}^{-3}$ ). The curve of HKUST-1-S approaches a plateau beyond the
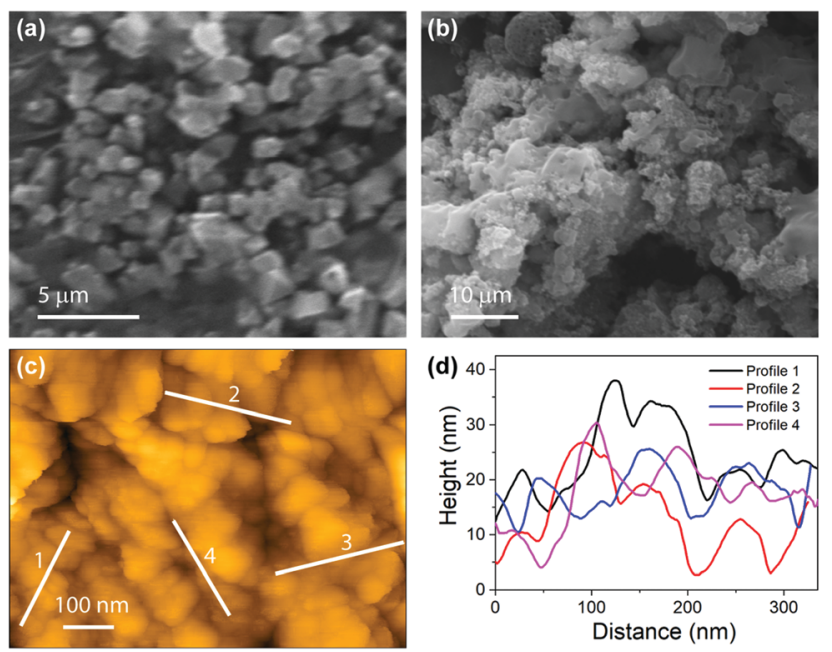

Fig. 1 Morphological study of the HKUST-1 MOF materials. SEM images of (a) HKUST-1-S crystals and (b) HKUST-1-T aggregates. (c) AFM topography image of the HKUST-1-T sample. (d) Height profiles of nanocrystals of HKUST-1-T extracted from the AFM topography (c).

application of about 3 ton load, but this effect is delayed in the case of the HKUST-1-T pellets. The densification data suggest that the occluded guest molecules within HKUST-1-T (Fig. 2(b)) could increase the mechanical stiffness of the porous framework, ${ }^{18,19}$ thus improving its resilience against mechanical deformation and collapse. The guest encapsulation and pelleting pressure-dependent systematic colour shift, from turquoise to dark blue (Fig. 2(a) inset), can be explained by the change in the refractive index $\left(n^{2}=k\right)$ which is also evident in the pressed powder pellets.

In order to confirm the presence of $\mathrm{NEt}_{3}$ guest molecules encapsulated in HKUST-1-T (Fig. 2(b)), we have employed a range of complementary techniques. Fig. 2(c) shows the distinctively different FTIR spectra of the two samples, wherein in addition to the common characteristic peaks of HKUST-1, a new vibrational band was detected at $1224 \mathrm{~cm}^{-1}$ assigned to the $\mathrm{C}-\mathrm{N}$ stretching mode of $\mathrm{NEt}_{3}$ (guest) found in HKUST-1-T. Additionally, the pressure-induced amorphization of the pellets was also evident from the systematic peak broadening of the characteristic vibrational modes in FTIR spectra as a function of pelleting pressure (Fig. S3, ESI $\dagger$ ). The amorphization of the HKUST-1 framework was corroborated by X-ray diffraction (Fig. S4 and S5, ESI $\dagger$ ), where the outcome of the FWHM analysis (Fig. S5, ESI $\dagger$ ) points to the pressure-induced amorphization of the framework. ${ }^{20,21}$ We note that the comparatively higher FWHM values of the compressed pellets of HKUST-1-T over HKUST-1-S can be ascribed to the prevalence of nanocrystals found in the former sample (Fig. 1(c)).

The encapsulation of $\mathrm{NEt}_{3}$ in the HKUST-1 framework was also investigated by $\mathrm{N}_{2}$ adsorption at $77 \mathrm{~K}$. The HKUST-1-T powder sample showed a BET surface area of $165 \mathrm{~m}^{2} \mathrm{~g}^{-1}$, which is appreciably lower than $1007 \mathrm{~m}^{2} \mathrm{~g}^{-1}$ determined for the HKUST-1-S powder. The adsorption data support the notion that the pores of HKUST-1-T are not fully accessible due to guest occlusion. From the nitrogen sorption isotherms in Fig. S6(a) (ESI $\dagger$ ), it can be surmised that HKUST-1-S and HKUST-1-T have 
(a)

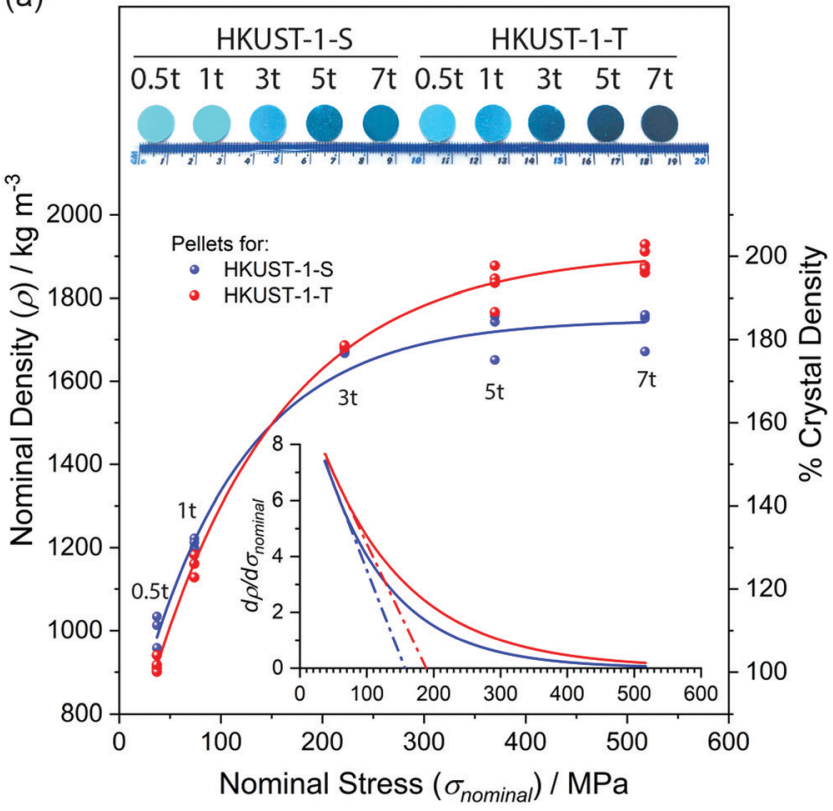

(b)

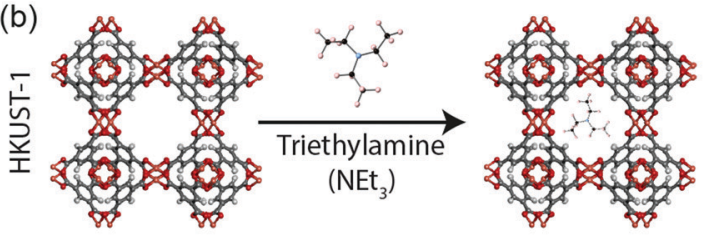

(c)

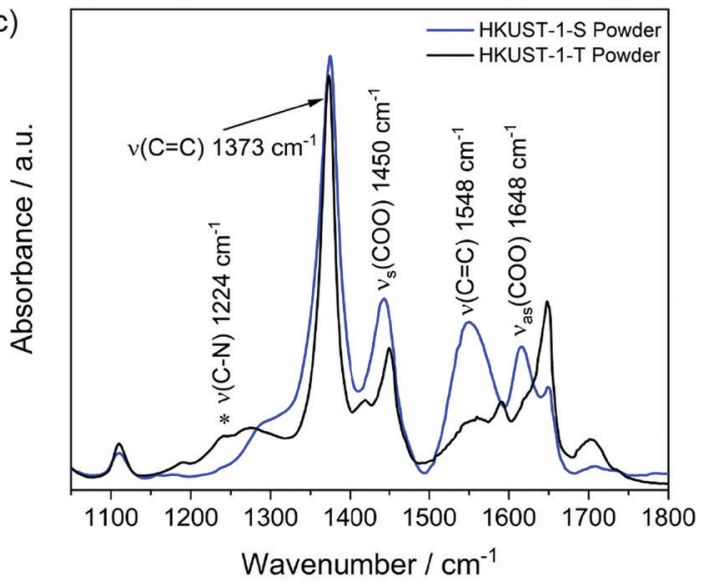

Fig. 2 (a) Density of HKUST-1 pellets as a function of the applied nominal pressure. A crystal density of $100 \%$ corresponds to the theoretical density of $948.9 \mathrm{~kg} \mathrm{~m}^{-3}$ calculated for a unit cell of an ideal HKUST-1 crystal. The top inset shows photographs of the pellets used in this study. The bottom inset shows the change in density with respect to the applied pelleting stress, where intercepts on the abscissa were used to estimate the yield strength of the compressed powder of HKUST-1-S and -T as $160 \mathrm{MPa}$ and $190 \mathrm{MPa}$, respectively. (b) Illustration of the encapsulation of triethylamine in the porous framework of HKUST-1. (c) FTIR spectra of HKUST-1-S and HKUST-1-T powders. The symmetric and asymmetric stretching modes are denoted as $\nu_{\mathrm{s}}$ and $\nu_{\text {as, }}$ respectively.

more pronounced microporosity and mesoporosity, respectively. Subsequently, we have confirmed this via pore size distribution analysis shown in Fig. S6(b) (ESI $\dagger$ ), where HKUST-1-S has predominantly micropores of $<2 \mathrm{~nm}$, whereas HKUST-1-T is composed of mesopores ranging from $\mathrm{ca}$. 2-20 nm. The mesoporosity of HKUST-1-T can again be attributed to the nanosized crystal aggregates shown in Fig. 1(b and c). Finally, TGA data in Fig. S6(c) (ESI $\dagger$ ) show that in contrast to HKUST-1-S, HKUST-1-T shows an additional weight loss from $170{ }^{\circ} \mathrm{C}$ to $280{ }^{\circ} \mathrm{C}$, which can be associated with the expulsion of $\mathrm{NEt}_{3}$. Subsequently we estimated an average of $1.5 \mathrm{NEt}_{3}$ guest molecule per unit cell of HKUST-1-T.

\subsection{Dielectric behaviour}

The activated HKUST-1 pellets were used to investigate the effects of pelleting pressure and guest encapsulation on the dielectric properties, as a function of temperature from $20^{\circ} \mathrm{C}$ to $100{ }^{\circ} \mathrm{C}$. All dielectric measurements were performed under vacuum and over the frequency range of $4 \mathrm{~Hz}$ to $1 \mathrm{MHz}$. The dielectric constant of a porous framework material, such as MOFs, depends on its effective density ${ }^{22,23}$ and its polarizability. ${ }^{7}$ In the proceeding section, it has been established that the pellet nominal density rises exponentially as $-\exp (-\sigma)$, with pelleting stress $\sigma$. Fig. 3(a) shows the real part of the dielectric constant $\left(\varepsilon^{\prime}\right)$ of HKUST-1-S pellets at $20{ }^{\circ} \mathrm{C}$, which increases from $2.79(0.5 t)$ to $4.46(7 t)$ at $1 \mathrm{MHz}$ frequency, i.e. $\varepsilon^{\prime}$ has risen by a factor of $\sim 1.6$. Clearly, this is caused by the pressure-induced densification of the porous framework and structural amorphization elucidated in Section 3.1. Notably, there is a marked jump of $\Delta \varepsilon>0.57$ between the $0.5 t$ and $1 t$ pellets followed by $\Delta \varepsilon>0.43$ between the $1 t$ and $3 t$ pellets, which could be linked to framework collapse when the applied stress approaches $\sim 160 \mathrm{MPa}$. Raising the temperature of the $0.5 t$ pellet from $20{ }^{\circ} \mathrm{C}$ to $100{ }^{\circ} \mathrm{C}$ has caused only a small increase of $\varepsilon^{\prime}$ from 2.79 to 2.86 , this finding suggests the absence of temperature-dependent dipole moments. ${ }^{24}$ Overall, we did not see a strong influence of temperature on the $\varepsilon^{\prime}$ and $\varepsilon^{\prime \prime}$ values of HKUST-1-S, although the densest $7 t$ pellet appears to be more sensitive to temperature change compared to the lower density pellets.

Turning to the guest-encapsulated samples, Fig. 3(b) shows that the dielectric response of HKUST-1-T is significantly different from that of HKUST-1-S, both in terms of the magnitude of $\varepsilon^{\prime}$ and its response to heating. Initially at $20{ }^{\circ} \mathrm{C}$, we determined that $\varepsilon^{\prime}$ of HKUST-1-T at $1 \mathrm{MHz}$ has increased from $2.81(0.5 t)$ to $6.48(7 t)$, i.e. a factor of 2.3 times higher due to the pelleting pressure. Upon compression, the $\mathrm{NEt}_{3}$ molecules (guest) may form weak interaction with the oxygen found at the Cu-paddle wheel of HKUST-1 (host) and contribute to the overall dielectric property in addition to the effect arising from framework densification, thus resulting in an increased of $\varepsilon^{\prime}$ value. A jump of $\Delta \varepsilon>1.34$ occurring between the $1 t$ and $3 t$ pellets of HKUST-1-T could be attributed to framework collapse, but this is detected at a stress of $\sim 190 \mathrm{MPa}$ which is relatively higher than HKUST-1-S ( 160 MPa, see Fig. 2(a) inset). This finding supports the hypothesis that the encapsulation of $\mathrm{NEt}_{3}$ guest molecules in HKUST-1-T improves its mechanical resilience. For the $0.5 t$ pellet, $\varepsilon^{\prime}$ at $1 \mathrm{MHz}$ increases from $2.81\left(20^{\circ} \mathrm{C}\right)$ to $3.16\left(100{ }^{\circ} \mathrm{C}\right)$. The rate of increase in the temperature-dependent $\varepsilon^{\prime}$ value is notably 

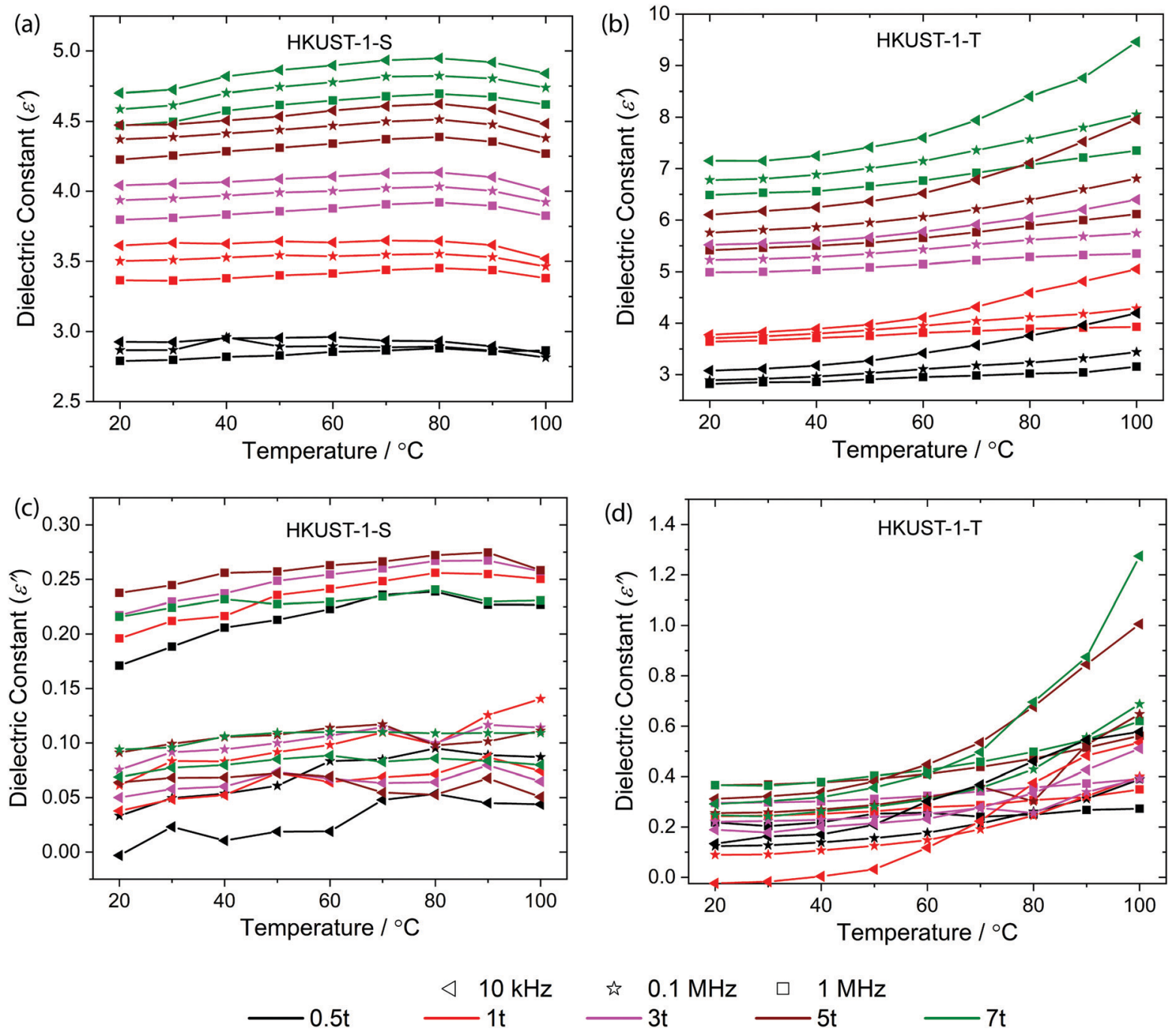

Fig. 3 Dielectric response as a function of temperature for the $0.5 t, 1 t, 3 t$, 5t, and $7 t$ pelleted samples of HKUST-1-S (left panels) and HKUST-1-T (right panels), all of which measured under vacuum conditions. (a and b) Real part of the dielectric constant $\varepsilon^{\prime}$. (c and d) Imaginary part of the dielectric constant $\varepsilon^{\prime \prime}$.

greater for samples obtained by higher pelleting pressure. For example, in the $7 t$ pellet, the value of $\varepsilon^{\prime}$ at the lower frequency of $10 \mathrm{kHz}$ went up drastically from 7.15 to 9.46 when the $20{ }^{\circ} \mathrm{C}$ sample was heated to $100{ }^{\circ} \mathrm{C}$. Our results suggest that the higher temperature will augment the dipole motion of the encapsulated $\mathrm{NEt}_{3}$ guests under an alternating electric field $(10 \mathrm{kHz}>0.1 \mathrm{MHz}>1 \mathrm{MHz})$, thereby giving a marked rise in the $\varepsilon^{\prime}$ and $\varepsilon^{\prime \prime}$ values with pelleting stress. The guest-encapsulated HKUST-1 thus exhibits a considerably stronger temperatureinduced dipole moment, which is different from that of the porous (guest-free) HKUST-1-S sample described above.

At higher frequency and temperature, the dipole moment cannot be kept up with the higher alternating field, resulting in energy loss and its contribution to polarization drops. This can be explained using the imaginary part of the dielectric constant $\left(\varepsilon^{\prime \prime}\right)$, which represents the dissipative losses. For the HKUST-1-S pellets, the dissipation of the porous framework was found to be notably greater at $1 \mathrm{MHz}$ frequency, and the loss gently increases with heating due to thermal perturbations. For the HKUST-1-T pellets, the steep increase in $\varepsilon^{\prime \prime}$ at the lower frequency of $10 \mathrm{kHz}$ in response to heating is associated with the loss induced by the encapsulated $\mathrm{NEt}_{3}$ molecules. This effect can be suppressed by elevating the frequency. The detailed frequency-dependent dielectric plots of both samples are presented in the ESI $\dagger$ (Fig. S9-S14).

We further investigated the dielectric properties of HKUST-1 under ambient conditions of $44 \% \mathrm{RH}$ (relative humidity), in the frequency range of $4 \mathrm{~Hz}$ to $8 \mathrm{MHz}$. The results are presented in Fig. S15-S17 of the ESI. $\dagger$ Interestingly, as shown in Fig. S15 (ESI $\dagger$ ), the pellets of HKUST-1-T show a relatively lower $\varepsilon^{\prime}$ value than HKUST-1-S, because the presence of $\mathrm{NEt}_{3}$ molecules occupying the pores of the former has lessened moisture uptake. The framework density and interaction between water and the $\mathrm{NEt}_{3}$ guest molecule play a major role in the HKUST-1-T pellets, both factors become more prevalent for higher pressure pellets resulting in the overall rise of the $\varepsilon^{\prime}$ values. In contrast, the dielectric response of the HKUST-1-S pellets is dominated by the adsorbed water molecules (because $\varepsilon^{\prime} \sim 80$ at $20{ }^{\circ} \mathrm{C}$ for water), whose occupancy will decline with higher pelleting pressure (giving reduced porosity), resulting 
in an overall reduction of dipole moments and thereby the lowering of the $\varepsilon^{\prime}$ values.

\subsection{Alternating current (AC) conductivity behaviour}

The frequency-dependent electrical properties of different HKUST-1 pellets were studied at increasing temperatures. Fig. 4(a) and (b) show the temperature-dependent conductivity of the HKUST-1 pellets. The slight increase in the conductivity of the HKUST-1-T pellets can be attributed to the $\mathrm{NEt}_{3}$ guest molecules present in the pores of the host framework. From the results it was deduced that the weak interaction of $\mathrm{NEt}_{3}$ with the framework caused by the pelleting pressure will increase the charge hopping probability and also decrease the percolation threshold causing a sudden increase in conductivity beyond the pelleting pressure of 1 ton, which was not observed in the HKUST-1-S pellets. Both the frequency and temperature affect the conductivity of HKUST-1 pellets, which can be observed for the individual pellet in the ESI $\dagger$ (Fig. S18 and S19).
Fig. 4(c and d) shows the real vs. imaginary part of the impedance of 0.5 and 7 ton pellets for a better understanding of the effect of pelleting pressure. The impedance data were fitted into a large arc using the resistance and two equivalent circuits connected in series (implemented in ZView software). The instrumental noise caused by the connections during measurement was represented by resistance $R_{1}$ in the circuit. In the second circuit, the constant phase element $\left(\mathrm{CPE}_{1}\right)$ and resistance $\left(R_{2}\right)$ are related to the bulk property of HKUST-1, whereas the constant phase element $\left(\mathrm{CPE}_{2}\right)$ and resistance $\left(R_{3}\right)$ of the third circuit are related to the interface between the electrode and the HKUST-1 pellets. The estimated capacitance value from the impedance curve was found to lie in the range of $\mathrm{pF} \mathrm{cm}^{-1}$, which can be attributed to the intrinsic property of the bulk material. The conductivity of pellets can be calculated using the equation $\sigma_{\mathrm{c}}=d /(R \times A)$, where $\sigma_{\mathrm{c}}$ is the conductivity, $d$ is the distance between electrodes, $A$ is the crosssection area and $R$ is the curve-fitted resistance. The calculated conductivity values of all the pellets are shown in Table S1 (ESI $\dagger$ ).
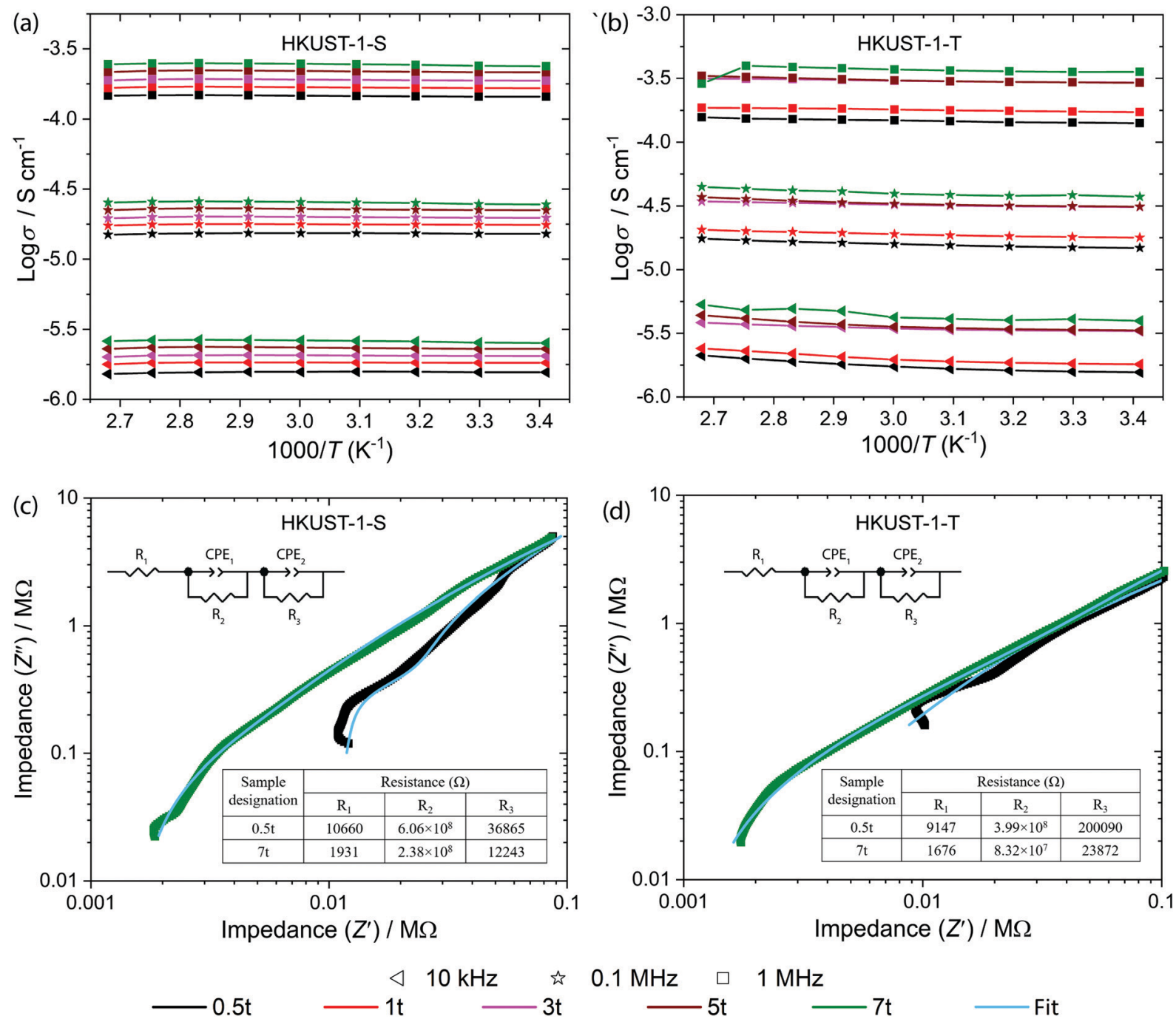

Fig. 4 AC conductivity as a function of temperature for (a) HKUST-1-S and (b) HKUST-1-T pellets prepared by different compression forces. Measurements were performed in a vacuum at three different frequencies of $10 \mathrm{kHz}, 0.1 \mathrm{MHz}$ and $1 \mathrm{MHz}$. Impedance plots of imaginary vs. real parts with the equivalent circuit for the 0.5 ton pellet at $20{ }^{\circ} \mathrm{C}$ and $100{ }^{\circ} \mathrm{C}$ for (c) HKUST-1-S and (d) HKUST-1-T. Note: CPE = constant phase element. 
The pattern of increase in the conductivity values of the HKUST-1-T pellets is consistent with the experimentally obtained dielectric and conductivity values. These conductivity results confirm the highly insulating nature of HKUST-1, which is central to the function of low- $k$ materials. All the fitted parameters of the real and imaginary parts of the impedance data are given in the ESI $\dagger$ (Fig. S24 and S25).

\section{Conclusions}

In summary, we presented a high-yield HKUST-1 MOF synthesis technique for low- $k$ and tuneable dielectric applications, where trimethylamine $\left(\mathrm{NEt}_{3}\right)$ guest molecules are confined in the porous

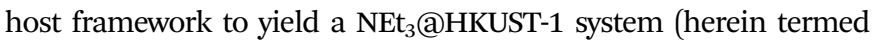
as HKUST-1-T). From thermogravimetric analysis, $\mathrm{NEt}_{3}$ was determined to be 1.5 molecules per formula unit of the host framework, thus resulting in a chemical formula of HKUST-1-T as $\left[\mathrm{Cu}_{3}(\mathrm{BTC})_{2}\right]$. $1.5\left[\mathrm{~N}\left(\mathrm{CH}_{2} \mathrm{CH}_{3}\right)_{3}\right]$. At $1 \mathrm{MHz}$ frequency, the $0.5 t$ pellet of the guestencapsulated material has a dielectric constant value of $\varepsilon^{\prime}=2.81$ at $20{ }^{\circ} \mathrm{C}$ and 3.14 at $100{ }^{\circ} \mathrm{C}$, which is an exceptional example of guest@MOF dielectrics at elevating temperatures, in contrast to the guest-free HKUST-1-S that exhibits a relatively smaller difference of $\varepsilon^{\prime}=2.79\left(20{ }^{\circ} \mathrm{C}\right)$ and $2.87\left(100{ }^{\circ} \mathrm{C}\right)$. Electrical impedance measurements and analysis revealed that the low-dielectric constant values are an intrinsic property of the host framework that can be modified by mechanical stress. Uniquely, we show that the guest-encapsulated material exhibits a broadly tuneable dielectric response through precise adjustments to parameters such as the frequency, temperature, and pelleting pressure. Moreover, the mechanical property of the open framework can be enhanced by guest encapsulation, where we show that the yield strength and structural stability of the compressed pellets can be improved by guest confinement. Collectively, the results demonstrate an innovative pathway for tailoring the physical behaviour of MOFs for dielectrics and other multifunctional devices.

\section{Conflicts of interest}

There are no conflicts to declare.

\section{Acknowledgements}

This research was funded by the EPSRC DTP-Samsung studentship award to A. S. B. J. C. T. thanks the European Research Council (ERC) Consolidator Grant through the grant agreement 771575 (PROMOFS) for funding the Guest@MOF research in the group. We are grateful to Dr Gavin Stenning and Mr Daniel Nye for providing access to the XRD facilities at the R53 Material Characterization Lab in ISIS Rutherford Appleton Laboratory, and to Dr James Taylor for performing the nitrogen adsorption measurements. We thank the Research Complex at Harwell (RCaH) for access to FTIR-ATR and TGA instruments. We are grateful to Carlos Cuadrado and Prof. Joaquin Silvestre who performed the NLDFT pore size distribution analysis.

\section{References}

1 O. Shekhah, J. Liu, R. A. Fischer and A. Woll, Chem. Soc. Rev., 2011, 40, 1081-1106.

2 G. Ferey, Chem. Soc. Rev., 2008, 37, 191-214.

3 M. R. Ryder and J. C. Tan, Mater. Sci. Technol., 2014, 30, 1598-1612.

4 Semiconductor Industry Association, 2013, (SEMATECH, Albany, NY, USA, 2013).

5 W. Volksen, R. D. Miller and G. Dubois, Chem. Rev., 2009, 110, 56-110.

6 K. Zagorodniy, G. Seifert and H. Hermann, Appl. Phys. Lett., 2010, 97, 251905.

7 M. R. Ryder, L. Dona, J. G. Vitillo and B. Civalleri, ChemPlusChem, 2018, 83, 308-316.

8 A. S. Babal, L. Dona, M. R. Ryder, K. Titov, A. K. Chaudhari, Z. Zeng, C. S. Kelley, M. D. Frogley, G. Cinque and B. Civalleri, J. Phys. Chem. C, 2019, 123, 29427-29435.

9 A. S. Babal, A. K. Chaudhari, H. H.-M. Yeung and J. C. Tan, Adv. Mater. Interfaces, 2020, 7, 2000408.

10 S. Mendiratta, M. Usman, T. T. Luo, S. F. Lee, Y. C. Lin and K. L. Lu, CrystEngComm, 2014, 16, 6309-6315.

11 M. Usman, C. H. Lee, D. S. Hung, S. F. Lee, C. C. Wang, T. T. Luo, L. Zhao, M. K. Wueg and K. L. Lu, J. Mater. Chem. C, 2014, 2, 3762-3768.

12 S. Mendiratta, M. Usman, C. C. Chang, Y. C. Lee, J. W. Chen, M. K. Wu, Y. C. Lin, C. P. Hsu and K. L. Lu, J. Mater. Chem. C, 2017, 5, 1508-1513.

13 E. Biemmi, S. Christian, N. Stock and T. Bein, Microporous Mesoporous Mater., 2009, 117, 111-117.

14 D. Zacher, J. Liu, K. Huber and R. A. Fischer, Chem. Commun., 2009, 1031-1033.

15 X. Mu, Y. Chen, E. Lester and T. Wu, Microporous Mesoporous Mater., 2018, 270, 249-257.

16 C. Xin, H. Zhan, X. Huang, H. Li, N. Zhao, F. Xiao, W. Wei and Y. Sun, RSC Adv., 2015, 5, 27901-27911.

17 A. K. Chaudhari, I. Han and J. C. Tan, Adv. Mater., 2015, 27, 4438-4446.

18 J. C. Tan, T. D. Bennett and A. K. Cheetham, Proc. Natl. Acad. Sci. U. S. A., 2010, 107, 9938-9943.

19 T. D. Bennett, J. Sotelo, J. C. Tan and S. A. Moggach, CrystEngComm, 2015, 17, 286-289.

20 T. Ungar, Scr. Mater., 2004, 51, 777-781.

21 T. D. Bennett and A. K. Cheetham, Acc. Chem. Res., 2014, 47, 1555-1562.

22 M. R. Ryder, Z. Zeng, K. Titov, Y. Sun, E. Mahdi, I. Flyagina, T. D. Bennett, B. Civalleri, C. S. Kelley and M. D. Frogley, J. Phys. Chem. Lett., 2018, 9, 2678-2684.

23 K. Titov, Z. X. Zeng, M. R. Ryder, A. K. Chaudhari, B. Civalleri, C. S. Kelley, M. D. Frogley, G. Cinque and J. C. Tan, J. Phys. Chem. Lett., 2017, 8, 5035-5040.

24 S. Eslava, L. Zhang, S. Esconjauregui, J. Yang, K. Vanstreels, M. R. Baklanov and E. Saiz, Chem. Mater., 2012, 25, 27-33. 